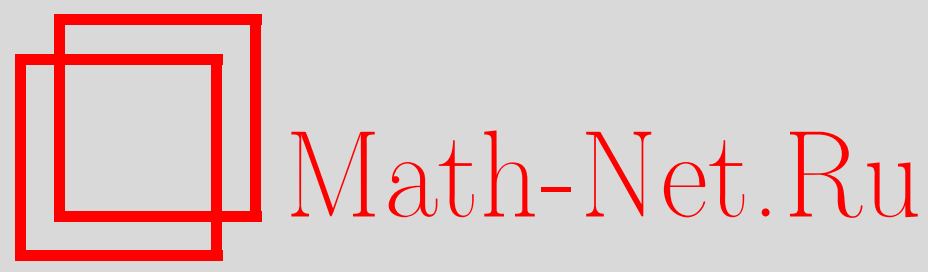

E. В. Соколов, Об аппроксимируемости конечными $p$ группами свободных произведений групп с нормальным объединением, Матем. заметки, 2005, том 78, выпуск 1, 125-131

DOI: https://doi.org/10.4213/mzm2569

Использование Общероссийского математического портала Math-Net.Ru подразумевает, что вы прочитали и согласны с пользовательским соглашением http://www . mathnet.ru/rus/agreement

Параметры загрузки:

IP : 54.198 .55 .26

26 апреля 2023 г., 18:09:55

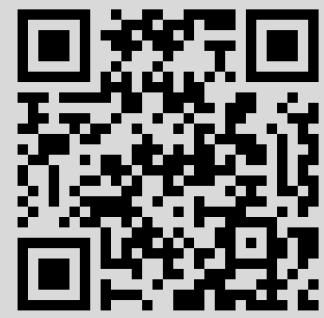




\title{
ОБ АППРОКСИМИРУЕМОСТИ КОНЕЧНЫМИ $p$-ГРУППАМИ СВОБОДНЫХ ПРОИЗВЕДЕНИЙ ГРУПП С НОРМАЛЬНЫМ ОБЪЕДИНЕНИЕМ
}

\author{
Е.В. Соколов
}

\begin{abstract}
Получено достаточное условие аппроксимируемости классом $\mathscr{F} p$ конечных $p$-групп свободного произведения $G=(A * B ; H)$ групп $A$ и $B$ с нормальной объединенной подгруппой $H$. С его помощью доказано, что если $A$ и $B$ представляют собой расширения $\mathscr{N}$-аппроксимируемых групп при помощи $\mathscr{F} p$-групп, где $\mathscr{N}$ обозначает класс конечно порожденных нильпотентных групп без кручения, и $H$ является нормальнй $p^{\prime}$-изолированной полициклической подгруппой, то группа $G$ аппроксимируется классом $\mathscr{F} p$, как только $\mathscr{F} p$-аппроксимируемой является факторгруппа $G / H^{p} H^{\prime}$.
\end{abstract}

Библиографоия: 10 названий.

1. Формулировка результатов. Напомним, что свободным произведением групп $A$ и $B$ с подгруппами $H \leqslant A$ и $K \leqslant B$, объединенными относительно изоморфизма $\varphi: H \rightarrow K$, назьвается групша $G=(A * B ; H=K, \varphi)$, задаваемая образуюшими и определяюшими соотношениями групा $A$ и $B$, а также всеми соотношениями вида $h=h \varphi$, где элемент $h$ пробегает подгрупу $H$. Как известно, групшы $A$ и $B$ вкладываются в группу $G$, поэтому их можно считать ее подгруппами. При этом подгруппы $H$ и $K$ оказьваются совпадающими, что позволяет записывать группу $G$ в виде $G=(A * B ; H)$ и называть ее свободньпм произведением групा $A$ и $B$ с объединенной подгруппой $H$ (считая изоморфизм $\varphi$ заданным). Введенные обозначения мы будем предполагать фиксированными на протяжении всей работы.

Напомним далее, что согласно общему определению А. И. Мальцева [1] подгруппа $Y$ некоторой групшы $X$ называется отделимой в классе груnп $\mathscr{K}$, или, короче, $\mathscr{K}$-отделимой, если для всякого элемента $x \in X \backslash Y$ сушествует гомоморфизм $\psi$ групшы $X$ на некоторую $\mathscr{K}$-групшу такой, что $x \psi \notin Y \psi$. Если мы возьмем здесь в качестве $Y$ единичную подгрупп, то получим определение аппроксимируемости группы $X$ в классе $\mathscr{K}$ ( $\mathscr{K}$-аппроксимируемости).

В настоящей работе речь идет об аптроксимируемости групы $G=(A * B ; H)$ классами $\mathscr{F}$ всех конечных групп и $\mathscr{F} p$ всех конечных $p$-групп. Традиционно эти свойства изучаются при тех или иных ограничениях, накладываемых на свободные множители $A, B$ и, в особенности, на объединенную подгруппу. Здесь рассматривается ситуация, когда подгруппа $H$ является “почти нормальной" в группе $G$, т.е. содержит подгруппу конечного индекса, нормальную в группах $A$ и $B$. 
Если $X$ - некоторая группа, то через $\Omega(X)$ и $\Omega_{p}(X)$ мы будем обозначать семейства всех нормальных подгрупп групшы $X$, имеющих в ней, соответственно, конечньй индекс и конечньй $p$-индекс. В работе [2] Р.Б. Д. Т. Олленби и Р.С. Грегорак показали, что обобщенное свободное произведение $G=(A * B ; H)$ двух почти полициклических груп аппроксимируется классом $\mathscr{F}$, как только семейство $\Omega(H)$ содержит подгрупу, нормальную во всей групше $G$. Повторяя почти дословно их рассуждение, мы приходим к следующему более общему результату.

Tеорема 1. Пусть группы $A$ и $B$ F-аппроксимируемы и все подгруппь из семейства $\Omega(H)$ являются $\mathscr{F}$-отделимыми в этих группах. Если кажсдая подгруппа $M \in \Omega(H)$ содержит подгруппу $L \in \Omega(H)$, нормальную в $G$, то группа $G$ $\mathscr{F}$-аппроксимируема.

Попытаемся теперь получить аналогичное условие апроксимируемости грушы $G$ классом $\mathscr{F} p$. Ситуация здесь оказьвается сложнее, поскольку в доказательстве теоремы 1 использовался тот факт, что свободное произведение $\mathscr{F}$-аппроксимируемых груп с конечным объединением всегда является $\mathscr{F}$-аппроксимируемойгруппой. Для класса $\mathscr{F} p$ это уже не так: даже свободное произведение двух конечных $p$-групш с нормальным объединением не обязано быть $\mathscr{F}$-аппроксимируемой групой, как показывает следующий

ПримеР. Пусть $H=\left\langle a ; a^{2}=1\right\rangle \times\left\langle b ; b^{2}=1\right\rangle, \alpha, \beta \in \operatorname{Aut}(H), a \alpha=b, b \alpha=a$, $a \beta=a b, b \beta=b$. Пусть также $A=\langle\alpha\rangle H$ и $B=\langle\beta\rangle H$ - подгрупшы голоморфа группы $H$ и $G=(A * B ; H)$ (изоморфизм $\varphi$ здесь является тождественньм отображением). Предположим, что группа $G \mathscr{F}_{2}$-аппроксимируема и, следовательно, существует гомоморфизм $\psi$ этой группы на конечную 2-группу, инъективный на множестве $A \cup B$.

Заметим, что произвольная конечная $p$-группа $F$ является нильпотентной и, как легко видеть, обладает некоторьм нормальным рядом, все факторы которого имеют порядок $p$. В силу теоремы Шрайера любой нормальный рядгрупшы $F$ может быть уплотнен до ряда с такими свойствами.

Отсюда вытекает, что подгруппа $H \psi$, нормальная в группе $G \psi$, должна содержать нормальную и, следовательно, центральную циклическую подгруппу порядка 2 . Непосредственная проверка показывает, что это невозможно и, таким образом, группа $G$ не является $\mathscr{F} 2$-аппроксимируемой.

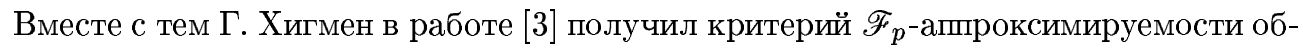
общенного свободного произведения двух $\mathscr{F} p$-груп, который без труда можно распространить на свободные произведения произвольных $\mathscr{F}$-аппроксимируемых групп с конечньм объединением. Поэтому всеприводимые ниже результаты будут сводить задачу к вопросу о том, является ли $\mathscr{F} p$-апшроксимируемым то или иное свободное произведение с конечной объединенной подгруппой.

Для удобства обозначим через $\Sigma_{p}(H)$ семейство всех таких подгрупп из $\Omega_{p}(H)$, которые нормальны в группах $A$ и $B$ (а следовательно, и в группе $G$ ).

Tеорема 2. Пусть группы $A$ и B $\mathscr{F}$-апnроксимируемы и все подгруппь из семейства $\Omega_{p}(H)$ являются $\mathscr{F}_{p}$-отделимыми в этих группах. Пусть также каждая подгруппа $M \in \Omega_{p}(H)$ coдержит подгруппу $L \in \Sigma_{p}(H)$. Eсли существует такая подгруппа $Q \in \Sigma_{p}(H)$, что подгруппа $Q^{p} Q^{\prime}$ имеет в ней конечный индекс и факторгруппа $G / Q^{p} Q^{\prime} \mathscr{F}$-аппроксимируема, то группа $G$ является $\mathscr{F}_{p}$-аппроксимируемой. 
Сформулированная теорема представляет собой обобщение основных результатов работы [4]. Применяя ее к свободньм произведениям с конечным объединением, мы получаем следующий критерий.

СлЕДСТВИЕ 3. Пусть группы $A$ u $B$ F्F -annроксимируемы, nодгрупnа $H$ конечна и нормальна в свободных множителях. Группа $G$ аппроксимируется клас-

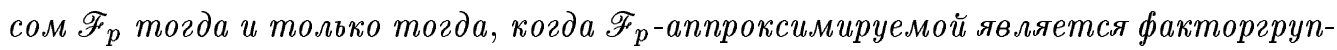
na $G / H^{p} H^{\prime}$.

Достаточность этого утверждения следует непосредственно из теоремы 2 , для доказательства же необходимости заметим, что если групп $G \mathscr{F} p$-апшроксимируема, то этим свойством будет обладать и ее факторгруппа по конечной подгруппе $H^{p} H^{\prime}$.

В действительности, условие теоремы 2 позволяет утверждать несколько большее, нежели $\mathscr{F}$-аппроксимируемость групшы $G$.

Пусть $X$ - некоторая группа; через $\Delta_{p}(X)$ мы будем обозначать семейство всех ее

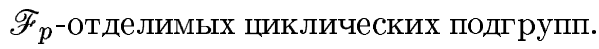

Легко видеть, что если подгруппа $Y$ принадлежит семейству $\Delta_{p}(X)$, то все корни $p^{\prime}$-степеней, извлекающиеся в групе $X$ из ее элементов, должны снова принадлежать $Y$ (здесь и далее $p^{\prime}$ обозначает множество всех простых чисел, не равных $p$ ). Подгруппу, обладающую этим свойством, назьвают $p^{\prime}$-изолированной в группе $X$.

Таким образом, если $X$ содержит хотя бы один элемент, порядок которого не является $p$-числом, то все циклические подгрупш группы $X$ уже заведомо не будут $\mathscr{F} p$-отделимьми. Поэтому в качестве аналога свойства $\mathscr{F}$-отделимости всех циклических подгрупп данной группы имеет смысл рассматривать утверждение об $\mathscr{F}$-отделимости всех ее $p^{\prime}$-изолированньх циклических подгруп. Меру отклонения группы $X$ от этого условия характеризует семейство $\bar{\Delta}_{p}(X)$ всех $p^{\prime}$-изолированных циклических подгрупп, не являющихся $\mathscr{F}$-отделимыми.

Обратимся теперь к группе $G=(A * B ; H)$. Очевидно, что если циклическая подгруппа группы $A$ не является $\mathscr{F} p$-отделимой в $A$, то она не будет $\mathscr{F} p$-отделимой и во всей группе $G$. Отсюда следует, что $\bar{\Delta}_{p}(A) \subseteq \bar{\Delta}_{p}(G)$ и точно так же $\bar{\Delta}_{p}(B) \subseteq \bar{\Delta}_{p}(G)$.

Мы будем говорить, что семейство $\Delta_{p}(G)$ максимально, если оно содержит все $p^{\prime}$-изолированные циклические подгруппы группы $G$, не сопряженные ни с какой подгруппой из семейства $\bar{\Delta}_{p}(A) \cup \bar{\Delta}_{p}(B)$. Имеет место

ТЕОрема 4. При выполнении условий теоремы 2 семейство $\Delta_{p}(G)$ является максимальным.

Отметим, что если групшы $A$ и $B \mathscr{F}$-аппроксимируемы, то максимальность семейства $\Delta_{p}(G)$ влечет за собой $\mathscr{F} p$-апшроксимируемость группы $G$ и, таким образом, теорема 4 представляет собой более сильное утверждение, нежели теорема 2.

В самом деле, если свободные множители являются $\mathscr{F}$-аппроксимируемыми группами, то семейства $\Delta_{p}(A)$ и $\Delta_{p}(B)$ содержат единичную подгрупшу. Ввиду максимальности эту подгруппу содержит и семейство $\Delta_{p}(G)$, поэтому группа $G$ также является

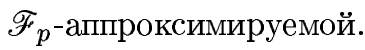

Оказьвается, что в случае конечной объединенной подгруппы имеет место и обратное утверждение; оно вытекает из теоремы 4 , если положить $Q=1$.

СлЕДСТВИЕ 5. Если подәруппа $H$ конечна и әруппа $G \mathscr{F}_{p}$-аппроксимируема, то семейство $\Delta_{p}(G)$ является максимальным. 
Применим теперь теорему 4 к обобщенным свободным произведениям групп, аппроксимируемых классом $\mathscr{N}$ всех конечно порожденных нильпотентных групп без кручения.

В работе [5] установлено, что в $\mathscr{N}$-аппоксимируемьг группах все $p^{\prime}$-изолированные полициклические подгруппы являются $\mathscr{F}$-отделимьми. Используя стандартное рассуждение из [1], нетрудно показать, что аналогичньй результат справедлив и для расширений групп указанного вида при помощи конечных $p$-групп [6].

ТЕОрема 6. Пусть группы $A$ и В представляют собой расширения $\mathcal{N}$-аппроксимируемых групп при помощи конечных р-групп, подгруппой, нормальной и $p^{\prime}$-изолированной в свободных множсителях. Если фак-

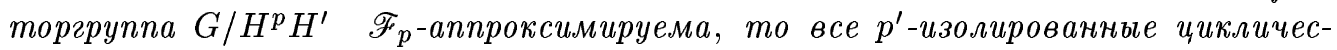

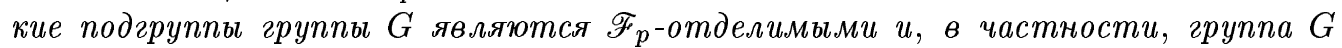

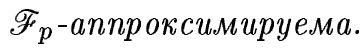

Эта теорема представляет собой обобщение ряда результатов Д.Н. Азарова [7] и Г. Кима и С. Танга [8] об $\mathscr{F}$-аппроксимируемости свободных произведений с циклическим объединением, а также одного из утверждений работы [4]. Для ее доказательства нам необходимо лишь проверить, что произвольная подгруппа $M \in \Omega_{p}(H)$ содержит некоторую подгруппу из семейства $\Sigma_{p}(H)$.

Обозначим через $n$ индекс подгруппы $M$ в $H$. Поскольку группа $H$ конечно порождена, она содержит лишь конечное число нормальных подгруп индекса $n$. Следовательно, их пересечение $L$ также имеет конечньй индекс в $H$, являющийся к тому же $p$-числом. Остается заметить, что подгруппа $L$ является характеристической в $H$ и потому нормальной в групше $G$.

2. Достаточные условия максимальности семейства $\Delta_{p}(G)$. В работе [9] было получено

ПРЕДЛОЖЕНИЕ 1. Пусть группы $A$ u $B$ F्ञ -аппроксимируемы, подгруппа $H$

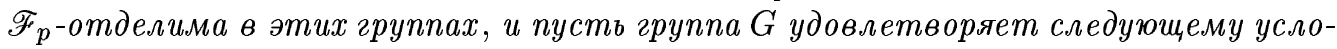
вию:

$$
\forall M \in \Omega_{p}(A) \quad \forall N \in \Omega_{p}(B) \quad \exists L \in \Omega_{p}(G) \quad(L \cap A \leqslant M \wedge L \cap B \leqslant N) .
$$

Тогда семейство $\Delta_{p}(G)$ является максимальным.

Сейчас мы покажем, что условие (1) этого предложения можно несколько ослабить.

Нормальный ряд конечной $p$-групшы будем называть главным, если все его факторы - циклические группы порядка $p$. С учетом этого определения упомянутый ранее

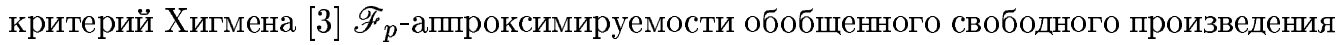
двух $\mathscr{F} p^{-г р у п п ~ в ь г л я д и т ~ с л е д у ю щ и м ~ о б р а з о м . ~}$

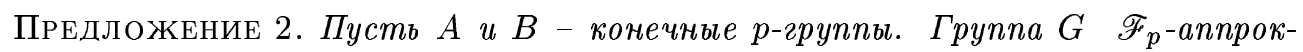
симируема тогда и только тогда, когда существуют главные ряды

$$
1=A_{0} \leqslant A_{1} \leqslant \cdots \leqslant A_{m}=A, \quad 1=B_{0} \leqslant B_{1} \leqslant \cdots \leqslant B_{n}=B
$$

әрупп $A$ и $B$ такие, что множества подәрупп $\left\{A_{i} \cap H\right\} u\left\{B_{j} \cap H\right\}$ coвпадают.

Рассматривая пример выше, мы уже отмечали, что каждая конечная $p$-группа $F$ обладает главным рядом и что произвольный нормальньй ряд групшы $F$ может быть уплотнен до главного. Из последнего замечания и предложения 2 вытекает 
ПРЕДЛОЖЕНИЕ 3. Пусть $A$ и $B$ - конечные $p$-группы, и пусть существуют нормальные ряды

$$
1=A_{0} \leqslant A_{1} \leqslant \cdots \leqslant A_{n}=A, \quad 1=B_{0} \leqslant B_{1} \leqslant \cdots \leqslant B_{n}=B
$$

групп $A$ и $B$ такие, что $A_{i} \cap H=B_{i} \cap H$ и порядки всех факторов $A_{i+1} \cap H / A_{i} \cap H$

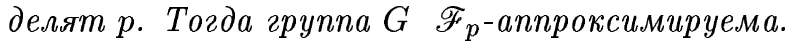

Действительно, поскольку порядок фактора $A_{i+1} \cap H / A_{i} \cap H$ делит $p$, для всякой подгрупшы $U$ такой, что $A_{i} \leqslant U \leqslant A_{i+1}$, пересечение $U \cap H$ должно совпадать либо с подгруппой $A_{i} \cap H$, либо с подгруппой $A_{i+1} \cap H$. Точно так же, если $B_{i} \leqslant V \leqslant B_{i+1}$, то $V \cap H=B_{i} \cap H$ или $V \cap H=B_{i+1} \cap H$. Таким образом, любые уплотнения исходных нормальных рядов групп $A$ и $B$ удовлетворяют условию предложения 2 и группа $G$ $\mathscr{F}$ р-аппроксимируема.

Теперь мы можем, наконец, привести несколько иную формулировку предложения 1.

ПРЕДЛОЖЕНИЕ 4. Пусть группы $A$ u $B$ F्ञ -anпроксимируемы, nодгруппа $H$

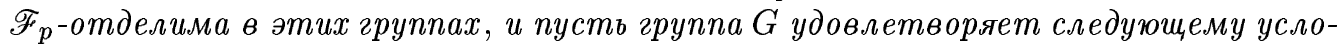
вию:

$$
\forall Q \in \Omega_{p}(H) \quad \exists L \in \Omega_{p}(G) \quad(L \cap H \leqslant Q) .
$$

Тогда семейство $\Delta_{p}(G)$ является максимальным.

Для доказательстванам в силу предложения 1 достаточно проверить, что для любых подгрупш $M \in \Omega_{p}(A)$ и $N \in \Omega_{p}(B)$ найдется подгрупша $U \in \Omega_{p}(G)$, удовлетворяющая соотношениям $U \cap A \leqslant M$ и $U \cap B \leqslant N$.

Обозначим пересечение $M \cap N \cap H$ через $Q$ и, пользуясь условием (2), выберем подгруппу $L \in \Omega_{p}(G)$ таким образом, чтобы $L \cap H \leqslant Q$.

Пусть $1=G_{0} / L \leqslant G_{1} / L \leqslant \cdots \leqslant G_{n} / L=G / L$ - некоторьй главньй ряд факторгруппы $G / L$. Положим $R_{0}=L \cap M, S_{0}=L \cap N$ и $R_{i}=G_{i} \cap A, S_{i}=G_{i} \cap B$, где $i=1,2, \ldots, n$. В результате мы получим неубьвающие последовательности нормальных подгрупп конечного $p$-индекса $R=R_{0} \leqslant R_{1} \leqslant \cdots \leqslant R_{n}=A$ и $S=S_{0} \leqslant S_{1} \leqslant \cdots \leqslant$ $S_{n}=B$, удовлетворяюшие соотношениям $R_{i} \cap H=S_{i} \cap H=L_{i} \cap H$ для любого $i \geqslant 0$.

Напомним, что через $K$ мы обозначали образ подгрупшы $H$ относительно изоморфизма $\varphi$. Условие $R \cap H=S \cap H$ означает, что $(R \cap H) \varphi=S \cap K$. Поэтому отображение $\varphi_{R, S}: H R / R \rightarrow K S / S$, ставящее в соответствие элементу $h R, h \in H$, элемент $(h \varphi) S$, корректно определено и является изоморфизмом подгрупп. Стало быть, мы можем построить группу

$$
G_{R, S}=\left(A / R * B / S ; H R / R=K S / S, \varphi_{R, S}\right) .
$$

Заметим еще, что естественные гомоморфизмы группы $A$ на $A / R$ и группы $B$ на $B / S$ продолжаемы до гомоморфизма $\rho_{R, S}$ групшы $G$ на групшу $G_{R, S}$.

Очевидно, что подгруппы $R_{i} \rho_{R, S}$ и $S_{i} \rho_{R, S}$ образуют в группах $A / R$ и $B / S$ нормальные ряды, удовлетворяющие условию предложения 3 . Поэтому группа $G_{R, S} \mathscr{F}_{\text {-ап- }}$ проксимируема, и мы можем найти подгруппу $U_{R, S} \in \Omega_{p}\left(G_{R, S}\right)$, тривиально пересекающуюся со свободными множителями.

Обозначая через $U$ прообраз подгрупш $U_{R, S}$ относительно гомоморфизма $\rho_{R, S}$, мы видим, что $U \cap A=R \leqslant M$ и $U \cap B=S \leqslant N$, т.е. подгрупша $U$ искомая. 
3. Доказательство теоремы 4. Если $X$ - некоторая группа и $Y$ - нормальная подгруппа группы $X$, то ограничение на подгруппу $Y$ произвольного внутреннего автоморфизма группы $X$ представляет собой некоторый автоморфизм группы $Y$. Подгруппу группы $\operatorname{Aut}(Y)$, составленную из всех таких автоморфизмов, мы будем обозначать $\operatorname{Aut}_{X}(Y)$.

Легко видеть, что отображение $\alpha_{Y}: X \rightarrow \operatorname{Aut}_{X}(Y)$, ставящее в соответствие каждому элементу $x \in X$ автоморфизм групшы $Y$, индуцированньй внутренним автоморфизмом $\hat{x}$ групшы $X$, гомоморфно. Ядро этого гомоморфизма совпадает с централизатором подгрупшы $Y$ в групе $X$.

Нетрудно показать также, что в $\mathscr{F}$-аппроксимируемой групе централизатор про-

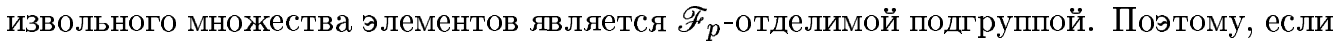
группа $G \mathscr{F}$-аппроксимируема, то для любой подгрупшы $Q \in \Sigma_{p}(H)$ группа $\operatorname{Aut}_{G}(Q)$ является $\mathscr{F} p$-апшроксимируемой. Хигмен в работе [3] показал, что наоборот, если $A$ и $B$ - конечные $p$-группы, подгруппа $H$ нормальна в свободных множителях $A, B$ и

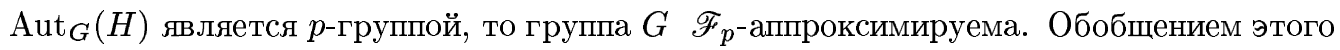
результата является

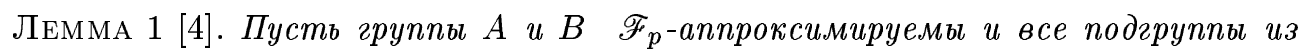

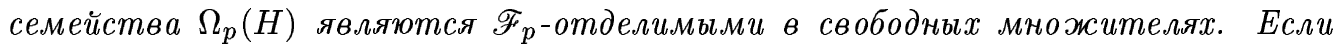
семейство $\Sigma_{p}(H)$ содерэсит такую подгруппу $Q$, что факторгруппа $G / Q \mathscr{F}_{\text {p-an- }}$ проксимируема и $\operatorname{Aut}_{G}(Q)$ является конечной p-группой, то әруппа $G \mathscr{F}_{p}$-anпроксимируема.

Если $X$ - некоторая группа, $Y$ - ее характеристическая подгруппа, то произвольный автоморфизм группы $X$ индуцирует некоторый автоморфизм групшы $Y$. Легко видеть, что отображение $\beta_{Y}: \operatorname{Aut}(X) \rightarrow \operatorname{Aut}(Y)$, переводящее элемент $\sigma \in \operatorname{Aut}(X)$ в его ограничение на подгруппу $Y$, гомоморфно. В работе Якушева [10] доказана

Лемма 2. Пусть $X$ - конечная $p$-группа, $\sigma \in \operatorname{Aut}(X)$. Порядок автоморфизма $\sigma$ является $p$-числом тогда и только тогда, когда р-числом является порядок индуиированного им автоморфизма факторгруппь $X / X^{p} X^{\prime}$.

Непосредственная проверка показьвает, что справедлива также

Лемма 3. Пусть $Y$ - нормальная подгруппа группь $X, Z$-характеристическая noдгpynna грyпnы $Y, \alpha_{Y}: X \rightarrow \operatorname{Aut}_{X}(Y)$ u $\alpha_{Y / Z}: X / Z \rightarrow \operatorname{Aut}_{X / Z}(Y / Z)$ - onpedeленные выше гомоморфизмы, $\varepsilon$ - естественный гомоморфизм группы $X$ на факmopгpynny $X / Z, \beta_{Z}: \operatorname{Aut}(Y) \rightarrow \operatorname{Aut}(Y / Z)$ - гомоморфизм индуиирования. Тогда следующая диаграмма коммутативна:

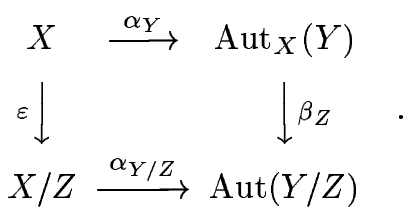

В частности, образ группы $\operatorname{Aut}_{X}(Y)$ относительно гомоморфизма $\beta_{Z}$ совпадает c noдzpynnoй $\mathrm{Aut}_{X / Z}(Y / Z)$.

Перейдем теперь собственно к доказательству теоремы. 
Пусть $M$ - произвольная подгруппа из семейства $\Omega_{p}(H)$. В силу предложения 4 нам достаточно показать, что существует подгруппа $N \in \Omega_{p}(G)$, удовлетворяющая соотношению $N \cap H \leqslant M$.

По условию подгруппа $M \cap Q$ содержит некоторую подгруппу $L \in \Sigma_{p}(H)$. Так как группы $G / Q$ и $G / Q^{p} Q^{\prime} L$ получаются из $G / Q^{p} Q^{\prime}$ факторизацией по конечным и, следо-

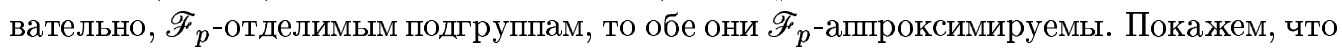
$\mathscr{F}$-аппроксимируемой является и факторгруппа $G_{1}=G / L$.

В силу леммы 1 нам достаточно убедиться в том, что (конечная) группа Aut $_{G_{1}}\left(Q_{1}\right)$, где $Q_{1}=Q / L$, является $p$-группой. Лемма 2 утверждает, что это верно тогда и только тогда, когда $p$-групшой является ее образ относительно гомоморфизма индуцирования $\beta_{T}$, где $T=Q_{1}^{p} Q_{1}^{\prime}=Q^{p} Q^{\prime} L / L$. Но в соответствии с леммой 3 этот образ совпадает с группой $\operatorname{Aut}_{G_{1} / T}\left(Q_{1} / T\right)$.

Факторгруппа $G_{1} / T$ изоморфна группе $G_{2}=G / Q^{p} Q^{\prime} L$, и этот изоморфизм индуцирует изоморфизм групп $\operatorname{Aut}_{G_{1} / T}\left(Q_{1} / T\right)$ и $\operatorname{Aut}_{G_{2}}\left(Q_{2}\right)$, где $Q_{2}=Q / Q^{p} Q^{\prime} L$. Так как

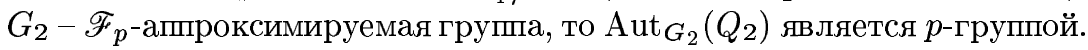

Таким образом, группа $G / L \mathscr{F}$-аппроксимируема и потому существует подгруппа $N / L \in \Omega_{p}(G / L)$ такая, что $N / L \cap H / L=1$. Отсюда $N \cap H=L \leqslant M$, и доказательство на этом закончено.

\section{СПИСОК ЦИТИРОВАННОЙ ЛИТЕРАТУРЫ}

[1] Мальцев А. И. О гомоморфизмах на конечные группы // Учен. зап. Ивановского гос. пед. ин-та. 1958. Т. 18. С. 49-60.

[2] Allenby R. B. J. T., Gregorac R. J. On locally extended residually finite groups // Lecture Notes in Math. 1973. V. 319. P. 9-17.

[3] Higman G. Amalgams of $p$-groups // J. Algebra. 1964. V. 1. P. 301-305.

[4] Соколов Е. В. Об аппроксимируемости конечными $p$-группами некоторых свободных произведений с объединенной подгруппой // Чебышевский сб. 2002. Т. 3. №1. С. 97-102.

[5] Соколов Е. В. Замечание об отделимости подгрупп в классе конечных $\pi$-групп // Матем. заметки. 2003. Т. 73. №6. С. 904-909.

[6] Соколов Е. В. Об отделимости циклических подгрупп в свободных произведениях двух групп с объединенной подгруппой // Деп. ВИНИТИ 12.07.2002 № 1325-В2002. Иваново: Ивановский гос. ун-т, 2002.

[7] Азаров Д. Н. О нильпотентной аппроксимируемости свободных произведений свободных групп с циклическим объединением // Матем. заметки. 1998. Т. 64. № 1. С. 3-8.

[8] Kim G., Tang C. Y. On generalized free products of residually finite $p$-groups // J. Algebra. 1998. V. 201. P. 317-327.

[9] Sokolov E. V. On the cyclic subgroup separability of free products of two groups with amalgamated subgroup // Lobachevskii J. Math. 2002. V. 11. P. 27-38.

[10] Якушев А. В. Аппроксимируемость конечными $p$-группами расщепляюшихся расширений групп // Научн. труды Ивановского гос. ун-та. Матем. 2000. № 3. С. 119-124.

Ивановский государственный университет

Поступило E-mail : ev-sokolov@yandex.ru 